\title{
OSCILLATION CRITERIA FOR NONLINEAR MATRIX DIFFERENTIAL EQUATIONS
}

\author{
KURT KREITH
}

Abstract. Oscillation criteria are established for nonlinear matrix differential equations of the form $\left[R(t) U^{\prime}\right]^{\prime}+F\left(t, U, U^{\prime}\right)=0$. These criteria are more general than some similar ones of $\mathrm{E}$. $\mathrm{C}$. Tomastik insofar as they do not require $F$ to be positive definite.

In [1] Tomastik derives oscillation criteria for nonlinear matrix differential equations of the form

$$
\left[R(t) U^{\prime}\right]^{\prime}+F\left(t, U, U^{\prime}\right) U=0
$$

under the hypothesis that the matrix $F$ is positive definite. The purpose of this note is to present an oscillation criterion for (1) which does not require such an assumption.

As in [1] we consider a "prepared" solution $U(t)$ of (1) satisfying

$$
U^{*}(t) R(t) U^{\prime}(t)=U^{* \prime}(t) R(t) U(t)
$$

and say that (1) is oscillatory on $[a, \infty)$ if the determinant of every prepared solution has arbitrarily large zeros. Our assumptions regarding the coefficient matrices are as in [1]. In particular, $R$ and $F$ are to be sufficiently regular, symmetric and real $n \times n$ matrices, and $R(t)$ is to be positive definite for all $t$.

The oscillation criterion to be derived below depends on a comparison of solutions of (1) and an equation of the same type,

$$
\left[P(t) V^{\prime}\right]^{\prime}+G\left(t, V, V^{\prime}\right) V=0 .
$$

LeMma 1. Let $U(t)$ be a prepared matrix solution of (1) such that $\operatorname{det} U(t) \neq 0$ on some interval $[b, c]$, and let $S(t)=R(t) U^{\prime}(t) U^{-1}(t)$. If $V(t)$ satisfies $(3)$ on $[b, c]$, then

$$
\begin{aligned}
{\left[V^{*} P V^{\prime}-V^{*} S V\right]_{l=b}^{l=c}=} & \int_{b}^{c} V^{*}(F-G) V d t+\int_{b}^{c} V^{* \prime}(P-R) V^{\prime} d t \\
& +\int_{b}^{c}\left(V^{\prime}-U^{\prime} U^{-1} V\right)^{*} R\left(V^{\prime}-U^{\prime} U^{-1} V\right) d t .
\end{aligned}
$$

Proof. If $\operatorname{det} U(t) \neq 0$, then a direct computation using (2) and the

Received by the editors May 8, 1969.

AMS 1969 subject classifications. Primary 3442, 3445.

Key words and phrases. Oscillation criteria, prepared solution, Picone-type identity, Sturm-Liouville equation, Leighton oscillation criterion. 
fact that $U^{-1 \prime}=-U^{-1} U^{\prime} U^{-1}$ yields the following Picone-type identity

$$
\begin{aligned}
\left(V^{*} P V^{\prime}-V^{*} R U^{\prime} U^{-1} V\right)^{\prime}= & V^{*}\left(P V^{\prime}\right)^{\prime}-V^{*}\left(R U^{\prime}\right)^{\prime} U^{-1} V+V^{* \prime}(P-R) V^{\prime} \\
& +\left(V^{\prime}-U^{\prime} U^{-1} V\right)^{*} R\left(V^{\prime}-U^{\prime} U^{-1} V\right) .
\end{aligned}
$$

Substituting (1) and (3) for the first two terms on the right side of this equation and integrating, (4) follows readily.

Lemma 2. Suppose $V(t)$ is a nontrivial solution of (3) satisfying

(i) $V^{*}(t)\left[F\left(t, U, U^{\prime}\right)-G\left(t, U, U^{\prime}\right)\right] V(t)$ is positive semidefinite for $b \leqq t \leqq c$ and all values of $U$ and $U^{\prime}$,

(ii) $V^{* \prime}(t)[P(t)-R(t)] V^{\prime}(t)$ is positive semidefinite for $b \leqq t \leqq c$,

(iii) $V(b)=V(c)=0$.

If $U(t)$ is a prepared solution of (1), then $\operatorname{det} U(t)$ has a zero in $[b, c]$.

Proof. If $\operatorname{det} U(t) \neq 0$ in $[b, c]$, then (4) holds and our hypotheses assure that the left side of (4) is 0 while each term on the right side of (4) is positive semidefinite. Furthermore, the last term on the right side of (4) is zero if and only if $V^{\prime}-U^{\prime} U^{-1} V \equiv 0$ on $[b, c]$, and this requires $V^{\prime}(b)=0$. By the uniqueness theorem for matrix systems, $V(b)=V^{\prime}(b)=0$ implies $V(t) \equiv 0$, contradicting the hypotheses and showing that det $U(t)=0$ for some $t$ in $[b, c]$.

Our oscillation criteria for (1) now follow easily by comparing (1) with the linear matrix equation

$$
\left[p(t) I V^{\prime}\right]^{\prime}+g(t) I V=0 .
$$

Let $J$ be a nonzero matrix with zeros and ones down the main diagonal and zeros elsewhere.

Theorem. If the Sturm-Liouville equation $\left(p(t) v^{\prime}\right)^{\prime}+g(t) v=0$ is oscillatory at $t=\infty$, and if for some real $a$ and some $J$

(i) $J\left[F\left(t, U, U^{\prime}\right)-g(t) I\right] J$ is positive semidefinite for $t \geqq a$ and all values of $U$ and $U^{\prime}$,

(ii) $J[p(t) I-R(t)] J$ is positive semidefinite for $t \geqq a$, then (1) is oscillatory on $[a, \infty)$.

Proof. Let $v(t)$ be a nontrivial solution of $\left(p v^{\prime}\right)^{\prime}+g v=0$ which is oscillatory at $\infty$ and define $V(t)=v(t) J$. Then $V(t)$ satisfies $\left(3^{\prime}\right)$, and we can find arbitrarily large pairs of numbers $(b, c)$ satisfying $c>b>a$ for which $V(b)=V(c)=0$. Furthermore

$$
V^{*}[F-g I] V=v^{2} J[F-g I] J \quad \text { and } \quad V^{* \prime}[p I-R] V^{\prime}=v^{\prime 2} J[p I-R] J
$$

so that conditions (i) and (ii) of Lemma 2 are satisfied on $[a, \infty)$. By Lemma 2 , equation (1) is oscillatory on $[a, \infty)$. 
In order to apply this Theorem, it is useful to recall the Leighton oscillation criterion: if

$$
\int^{\infty} \frac{1}{p_{1}(t)} d t=\int^{\infty} g_{1}(t) d t=\infty,
$$

then $\left(p_{1} v^{\prime}\right)^{\prime}+g_{1} v=0$ is oscillatory at $t=\infty$. Consider now the system (1) where $n=2$ and

$$
0<R(t) \leqq\left(\begin{array}{lr}
p_{1}(t) & 0 \\
0 & p_{2}(t)
\end{array}\right), \quad F\left(t, U, U^{\prime}\right) \geqq\left(\begin{array}{ll}
g_{1}(t) & 0 \\
0 & g_{2}(t)
\end{array}\right) .
$$

According to the Theorem with

$$
J=\left(\begin{array}{ll}
1 & 0 \\
0 & 0
\end{array}\right)
$$

if (5) is satisfied then (1) is oscillatory. This result does not follow from [1].

\section{REFERENCES}

1. E. C. Tomastik, Oscillation of nonlinear matrix differential equations of second order, Proc. Amer. Math. Soc. 19 (1968), 1427-1431. MR 38 \#372.

2. E. L. Ince, Ordinary differential equations, Longmans, Green, London, 1926; reprint, Dover, New York, 1944. MR 6, 65.

University of California, Davis, California 95616 